\title{
CALIDAD EN REVISIONES SISTEMÁTICAS DE EVALUACIONES ECONÓMICAS DE TECNOLOGÍAS \\ EN SALUD
}

Carolina Castañeda Guerrero

Universidad Nacional de Colombia

Colombia

Fernando De la Hoz Restrepo Universidad Nacional de Colombia Colombia

Nelson Alvis Guzmán Universidad de Cartagena Colombia 
Panorama Económico, Vol. 27 - No. 3 (Julio - Septiembre de 2019), pp. 581-597

Carolina Castañeda Guerrero.

Fernando De la Hoz Restrepo.

Nelson Alvis Guzmán

\title{
Calidad en revisiones sistemáticas de evaluaciones económicas de tecnologías en salud
}

\begin{abstract}
Resumen
La toma de decisiones en salud tanto desde el punto de vista clínico como administrativo, requiere entre otros aspectos, fundamentarse tanto en la mejor evidencia producto de investigación de las tecnologías sanitarias como en el mejor uso de los escasos recursos económicos con los que usualmente se cuenta. En el ámbito clínico, las revisiones sistemáticas de ensayos clínicos controlados y aleatorizados, aportan información valiosa al sintetizar la mejor evidencia. Por otro lado, las evaluaciones económicas de tecnologías sanitarias son útiles al proporcionar información comparada entre los costos de dicha tecnología, por unidad de desenlace de la enfermedad en términos usualmente de efectividad o de utilidad. Realizar revisiones sistemáticas de evaluaciones económicas de tecnologías sanitarias en principio podría considerarse una muy buena herramienta para toma de decisiones, sin embargo, es importante considerar que las evaluaciones económicas pueden presentar debilidades metodológicas que limitarían su calidad.
\end{abstract}

Las revisiones sistemáticas de ensayos clínicos cuentan con metodologías claras para realizarlas, incluyendo la evaluación de riesgo de sesgo y calidad tanto de los estudios incluidos, como de la misma revisión. En el caso de las evaluaciones económicas aún existe heterogeneidad en la metodología y falta de estandarización en la forma de evaluar su calidad, lo cual afecta el desarrollo de revisiones sistemáticas de evaluaciones económicas. Sin embargo, en la presente revisión se identifican herramientas que pueden servir para evaluar calidad y riesgo de sesgo de evaluaciones económicas, así como también de las mismas revisiones.

Palabras clave: Tecnologías sanitarias, tecnologías en salud, revisiones sistemáticas, evaluaciones económicas, calidad, evaluación calidad

\section{Qualité dans les revues systématiques des évaluations économiques des technologies de la santé}

\section{Résumé:}

La prise de décision dans le domaine de la santé, tant du point de vue clinique qu'administratif, exige, entre autres aspects, de s'appuyer sur les meilleures preuves de la recherche en technologie de la santé et sur la meilleure utilisation des rares ressources économiques avec généralement qu'ils ont. Dans le contexte clinique, les examens systématiques d'essais cliniques contrôlés et randomisés fournissent des informations précieuses en résumant les meilleures preuves. D'autre part, les évaluations économiques des technologies de la santé sont utiles pour fournir des informations comparatives entre les coûts de ladite technologie, par unité de résultat de maladie en termes d'efficacité ou de valeur d'utilité. L'examen systématique des évaluations économiques des technologies de la santé pourrait en principe être considéré comme un très bon outil de prise de décision; Cependant, il est important de garder à l'esprit que les évaluations économiques peuvent présenter des faiblesses méthodologiques qui limiteraient leur qualité.

Les revues systématiques d'essais cliniques ont des méthodologies claires pour les réaliser, comprenant l'évaluation du risque de biais et de la qualité des études incluses et la même revue. Dans le cas des évaluations économiques, il existe toujours une hétérogénéité dans la méthodologie et un manque de normalisation dans la manière d'évaluer sa qualité, ce qui affecte le développement d'examens systématiques des évaluations économiques. Cependant, cet examen identifie des outils pouvant être utilisés pour évaluer la qualité et le risque de biais dans les évaluations économiques, ainsi que les mêmes examens.

Mots clés: Technologies de la santé, revue systématique, évaluation économique, qualité, évaluation qualité 
Panorama Económico, Vol. 27 - No. 3 (Julio - Septiembre de 2019), pp. 581-597

Carolina Castañeda Guerrero.

Fernando De la Hoz Restrepo.

Nelson Alvis Guzmán

\title{
Quality in systematic reviews of economic evaluations of health technologies
}

\begin{abstract}
The decision making in the field of health both from a clinical and administrative point of view requires, among other aspects, to be based both on the best research evidence of health technologies and on the best use of the scarce economic resources with which it is usually had. In the clinical setting, systematic reviews of controlled and randomized clinical trials provide valuable information by summarizing the best evidence. On the other hand, economic evaluations of health technologies are useful in providing comparative information between the costs of said technology, per unit of outcome of the disease in terms usually of effectiveness or utility value. Conducting systematic reviews of economic evaluations of health technologies could in principle be considered a very good tool for decision making, however, it is important to consider that economic evaluations may present methodological weaknesses that would limit their quality.
\end{abstract}

Systematic reviews of clinical trials have clear methodologies to perform them, including the risk assessment of bias and quality of both the included studies and the same review. In the case of economic evaluations, there is still heterogeneity in the methodology and lack of standardization in the way of evaluating its quality, which affects the development of systematic reviews of economic evaluations. However, this review identifies tools that can be used to evaluate quality and risk of bias in economic evaluations, as well as the same reviews.

Key words: Health technologies, systematic review, economic evaluation, quality, quality assessment 


\title{
Calidad en revisiones sistemáticas de evaluaciones económicas de tecnologías en salud
}

\author{
Carolina Castañeda Guerrero \\ Universidad Nacional de Colombia, Colombia
}

INFORMACIÓN DEL ARTÍCULO

Recepción del artículo: 15/03/2019

Concepto de evaluación: 30/05/2019

Aceptación del artículo: 15/06/2019
Fernando De la Hoz Restrepo Universidad Nacional de Colombia, Colombia

Nelson Alvis Guzmán Universidad de Cartagena, Colombia

\section{INTRODUCCIÓN}

La investigación de tecnologías en salud produce continuamente múltiples publicaciones de estudios individuales con variabilidad metodológica que puede presentar debilidades, sesgos, ser dependientes de contexto o incluso obtener conclusiones contradictorias, lo cual puede producir confusión en la toma de decisiones, tanto para la práctica clínica como en administración sanitaria o políticas en salud. Por tanto, resumir hallazgos de la mejor evidencia de los estudios individuales, mediante métodos explícitos, preestablecidos y reproducibles, que es característico de las revisiones sistemáticas, puede proporcionar estimaciones confiables sobre las tecnologías en salud, facilitando decisiones fundamentadas en las mejores prácticas originadas en la investigación disponible (Akers J et al., 2009).

La toma de decisiones en administración sanitaria o políticas en salud se afecta adicionalmente por restricciones presupuestales que obligan a la búsqueda de asignación eficiente de los recursos en pro de la sostenibilidad de los sistemas de salud (Luhnen, Prediger, Neugebauer, \& Mathes, 2019). Por tanto, las consideraciones económicas de las tecnologías en salud toman relevancia si pueden ser comparadas con sus efectos, lo que resulta posible mediante las evaluaciones económicas. Sin embargo, dichos estudios pueden presentar limitaciones por su variabilidad metodológica e incertidumbre en las fuentes de información, lo que puede afectar su calidad (M. Drummond et al., 2009).

Las revisiones sistemáticas de ensayos clínicos controlados cuentan con metodologías rigurosas para su desarrollo, incluyendo sistemas de evaluación y gradación de evidencia, de reporte, evaluación metodológica e incluso de las mismas revisiones (Higgins \& 
Green, 2011). Para otro tipo de estudios, como las evaluaciones económicas, se cuenta con algunas herramientas que tienen un menor grado de desarrollo y de estandarización (Langer, 2012).

En el presente artículo se realizó una revisión para identificar herramientas útiles en evaluación de calidad tanto de revisiones sistemáticas, como de evaluaciones económicas, así como también de las herramientas que se podrían aplicar en las revisiones sistemáticas de las evaluaciones económicas, lo cual puede promover que estos estudios se realicen con mayor rigurosidad metodológica y sus resultados sean más confiables y útiles para apoyar el proceso de toma de decisiones en salud.

En primera instancia es importante definir las tecnologías en salud, las cuales según información de la Red Internacional de Agencias de Evaluación de Tecnologías en Salud (INHATA), se definen como "una intervención que se puede utilizar para promover la salud, prevenir, diagnosticar o tratar enfermedades agudas o crónicas, o para rehabilitación. Esto incluye productos farmacéuticos, dispositivos, procedimientos y sistemas organizativos utilizados en la atención médica" (INHATA)

\section{¿QUÉ ES UNA REVISIÓN SISTEMÁTICA EN EL CAMPO SANITARIO?}

Las revisiones sistemáticas surgieron ante la multiplicidad de investigación en salud y la falta de integración de resultados de eficacia de intervenciones, en resúmenes metodológicamente estructurados que pudieran mostrar acumulación de conocimiento de estudios con fundamentos válidos (Cochrane Website). Según el manual Cochrane de revisiones sistemáticas de intervenciones versión 5.1.0, las revisiones sistemáticas buscan responder una pregunta específica de investigación, reuniendo toda la evidencia empírica que cumpla unos criterios de elegibilidad previamente establecidos, se debe evaluar la validez de los resultados de dicha evidencia, minimizar errores sistemáticos (sesgos), para aportar resultados confiables, que permitan concluir y tomar decisiones (Higgins \& Green, 2011).

Las revisiones sistemáticas pueden contener meta-análisis, los cuales resultan de la aplicación de métodos estadísticos que resumen o combinan los resultados de los estudios incluidos, obteniendo información más precisa de los efectos sobre la atención sanitaria que la originada en los estudios independientes, siempre y cuando estos estudios sean homogéneos y sin riesgo de sesgo(Higgins \& Green, 2011).

Las revisiones sistemáticas inicialmente fueron creadas para resumir los hallazgos de ensayos clínicos controlados y aleatorizados, considerándose tradicionalmente como la más alta jerarquía en la pirámide de la medicina basada en la evidencia (Howick et al., 2011; Manterola, Asenjo-Lobos, \& Otzen, 2014; Sackett, Rosenberg, Gray, Haynes, \& Richardson, 1996), para algunos están primero las que realizan meta-análisis (SIGN, 2011), sin embargo, también es cuestionada esta posición al considerarse que las revisiones y meta-análisis no se encuentran exentos de incertidumbre, errores o sesgos (Borgerson, 2009; Herner, 2019; Murad, Asi, Alsawas, \& Alahdab, 2016). Posteriormente se han realizado revisiones sistemáticas de estudios observacionales, e incluso de evaluaciones económicas, encontrándose en aumento estas últimas (Luhnen et al., 2019), aunque han sido vistas con mayor reticencia. 
La calidad de la revisión sistemática depende de la calidad de los estudios incluidos, y del seguimiento estricto de los lineamientos preestablecidos para realizar estas revisiones. En el manual Cochrane para evaluar la calidad metodológica de los estudios no recomiendan el uso de escalas con puntuación resumen, para evaluar la calidad metodológica de los estudios, porque consideran que pueden existir criterios en las escalas que no se relacionan directamente con la validez interna de los estudios. Recomendando usar herramientas basadas en dominios que proporcionen descripción y valoración para cada ítem, pero no describen ninguna en particular. Sin embargo, consideran que, en lugar de evaluar calidad como los estándares más altos en la realización del estudio, se debe evaluar la validez de los resultados de los estudios mediante, por ejemplo, la valoración del riesgo de sesgo de los estudios individuales (Higgins \& Green, 2011).

Para evaluar la calidad de la evidencia, en este manual adoptan el sistema del grupo de trabajo GRADE "Grades of Recommendation, Assessment, Development and Evaluation" el cual define esta calidad como la medida en que se puede confiar que una estimación del efecto es correcta. Este sistema considera cuatro elementos clave, primero: el diseño del estudio: enfocándose en ensayos clínicos controlados aleatorizados y estudios observacionales; segundo: la calidad del estudio, que relacionan a los métodos detallados y a la ejecución de los estudios, lo que en el manual Cochrane consideran como riesgo de sesgo dentro de los estudios y lo incluyen en calidad metodológica. Un tercer elemento que denominan consistencia, se relaciona a la similitud de las estimaciones del efecto entre los estudios, y un cuarto elemento que llaman direccionalidad, corresponde a la medida en que la población, intervenciones y medidas de resultado son similares a los de interés (Atkins et al., 2004).

Además de la calidad de los estudios es importante la de la revisión, para esto son reconocidas dos herramientas, la declaración PRISMA -Preferred Reporting Items for Systematic reviews and Meta-Analyses- (Liberati et al., 2009) y la AMSTAR -Assessment of multiple systematic reviews- (Shea et al., 2007). PRISMA fue concebida para mejorar la claridad y transparencia en el reporte de las revisiones sistemáticas para su publicación y se originó como una actualización y ampliación de la lista QUOROM -Quality of Reporting of Meta-analysis- publicada en 1999 (Urrutia \& Bonfill, 2010).

PRISMA a diferencia de QUOROM incluye no solo meta-análisis, sino revisiones sistemáticas, amplía este listado de 18 a 27 ítems, considera un diagrama de flujo de resultados de búsqueda más detallado, el cual incluso fue incluido en el Manual Cochrane 5.1.0., se focaliza en la pregunta de investigación, la existencia de un protocolo previo y considera importante incluir la descripción de métodos utilizados para la evaluación de riesgo de sesgo de cada estudio revisado, sesgo entre los estudios y en los resultados (Liberati et al., 2009).

Como PRISMA se enfoca más en el reporte de la revisión, la otra herramienta mencionada es AMSTAR, la cual fue estructurada para evaluar la metodología de las revisiones sistemáticas y consta de 11 dominios, fue aplicada a 151 revisiones, logrando una herramienta con validez de cara y de contenido e incluso con validez de constructo (Shea et al., 2009). Entre los 11 dominios se encuentra, la evidencia de diseño a priori de la revisión, duplicación de revisores en selección de estudios y extracción de datos, búsqueda exhaustiva de literatura, estudios incluidos y excluidos, estado de publicación como criterio de inclusión, características de los estudios, evaluación de calidad científica de los estudios incluidos, combinación adecuada de hallazgos, probabilidad de sesgo de publicación y conflicto de intereses. 
Recientemente fue publicado por los mismos desarrolladores una actualización de esta herramienta llamada AMSTAR 2, a la cual no realizaron validación, considerando que de los 11 dominios de la herramienta original validada, se conservaron 10, aunque presenta algunos cambios resumiendo unos dominios y agregando otros como; manejo de riesgo de sesgo en síntesis de la evidencia, causas e importancia de la heterogeneidad y justificación de la selección de diseños de estudio. Sin embargo, enfatizan en que la lista es una sugerencia y los evaluadores pueden agregar o sustituir dominios críticos (Shea et al., 2017).

AMSTAR ha sido frecuentemente recomendada por diferentes grupos de investigación y se ha mencionado que la Colaboración Cochrane, podría considerarla en la actualización de su manual, para evaluar revisión de revisiones sistemáticas de intervenciones de cuidado en salud (Pollock, Fernandes, Becker, Featherstone, \& Hartling, 2016; Pollock, Fernandes, \& Hartling, 2017). Así como también, en conjunto con PRISMA, pueden ser útiles para evaluar tanto la metodología como el reporte de las revisiones que se incluyen en estas revisiones (Smith, Devane, Begley, \& Clarke, 2011). En el manual Cochrane 5.1.0, la revisión de revisiones son denominadas como "Overview", en el capítulo 22, y las definen como revisiones "para compilar la evidencia de múltiples revisiones sistemáticas de intervenciones en un documento accesible y utilizable (Higgins \& Green, 2011).

Con respecto a riesgo de sesgo, en AMSTAR, explícitamente mencionan si se valoró la probabilidad de sesgo de publicación y en AMSTAR 2, preguntan si usaron una técnica satisfactoria para evaluar el riesgo de sesgo de los estudios individuales incluidos, el impacto potencial de este riesgo sobre los resultados, si se incluye en la discusión y si se investiga el sesgo de publicación y su impacto en los resultados de la revisión.

Un dominio de AMSTAR, se refiere a evaluar y documentar la calidad científica de los estudios incluidos, y otro dominio si esta calidad se usa de manera adecuada al formular las conclusiones, pero la definición de esa calidad científica no es clara, es como si se refiriera solamente al protocolo previo, al requerir métodos a priori. En AMSTAR 2, no explicitan calidad, en lugar de ello especifican si se usó técnica satisfactoria para evaluar riesgo de sesgo en los estudios individuales y si se consideró en los resultados de la revisión. En AMSTAR 2, los desarrolladores no promueven la generación de un puntaje total, pero si la evaluación de dominios críticos y la valoración de la confianza general en los resultados de la revisión.

PRISMA a pesar de ser una lista centrada en revisiones sistemáticas de reporte de ensayos clínicos aleatorios, en ella se contemplan enunciados relevantes para reconocer limitaciones de información, como para fomentar transparencia y comprensión de los procesos adoptados en revisiones sistemáticas de diferentes tipos de estudios. De igual manera AMSTAR fue validado en revisiones sistemáticas de ensayos clínicos controlados, pero los desarrolladores consideraron podía ser adecuado para revisiones de otro tipo de estudios e incluso en revisión de revisiones (Pieper, Koensgen, Breuing, Ge, \& Wegewitz, 2018).

Sin embargo, desde otro punto de vista, algunos consideran que, las listas de verificación de PRISMA y AMSTAR están destinadas a evaluar el informe y la conducta de las revisiones pero que su uso no garantiza la capacidad de una revisión para alcanzar una comprensión correcta de la relevancia y credibilidad de los hallazgos (Thulliez, Angoulvant, Pisella, \& Bejan-Angoulvant, 2018). 


\section{¿QUÉ ES UNA EVALUACIÓN ECONÓMICA DE TECNOLOGÍAS EN SALUD?}

En el campo sanitario la metodología de las evaluaciones económicas, contempla un análisis comparativo de acciones o tecnologías alternativas en términos de sus costos y consecuencias, buscando la que presente mejor relación entre los costos asociados al uso de las mismas y sus resultados, los cuales pueden ser medidos como efectividad en desenlaces (evaluados desde la epidemiología clásica en unidades naturales como mortalidad o morbilidad evitada, etc), beneficios económicos, o en medidas de utilidad (años de vida ajustados por discapacidad -AVAD, años de vida ajustados por calidad AVAC, etc), definiéndose como estudios de costo efectividad, costo beneficio o costo utilidad, respectivamente, sin embargo en estudios de costo efectividad también pueden ser considerados en los desenlaces medidas como AVACs o AVADs (Soto-Alvarez, 2012).

En salud son muy utilizadas las evaluaciones de costo efectividad y de costo-utilidad, dado que las evaluaciones de costo-beneficio, no son muy aceptadas en el medio, porque valorar desenlaces en salud en términos monetarios ha conllevado a múltiples controversias (Drummond MF, 2015). Las evaluaciones económicas son útiles para establecer prioridades y guiar decisiones sobre la mejor elección de tecnologías en salud, ya sean nuevas o la combinación de existentes para que la asignación de recursos sea más eficiente.

Se ha considerado que las evaluaciones económicas se fundamentan en la teoría económica del bienestar, basada en el utilitarismo de Jeremy Bentham y el enfoque paretiano de eficiencia económica ( $\mathrm{Ng}, 2004)$. En el campo sanitario mediante las evaluaciones económicas se pretende analizar la contribución al bienestar de la población de una tecnología ó programa en salud, utilizando los recursos de la forma más eficiente (Briggs A, 2007). Sin embargo, para el caso de las evaluaciones económicas de costo efectividad, se ha propuesto la extensión de dicha teoría, definiéndola como extra-bienestarismo, argumentando que la utilidad no es la única función relevante en el bienestar social, sino que la salud es el desenlace más importante y que las fuentes de valoración de los principales resultados, pueden diferir en la salud (Brouwer, Culyer, van Exel, \& Rutten, 2008; Gray, 2011).

Para realizar evaluaciones económicas, según la Sociedad profesional de Economía de la Salud e investigación de resultados -ISPOR, varios países poseen unas guías o políticas oficiales reconocidas para toma de decisiones de cuidado en salud, otros países cuentan con guías o recomendaciones dadas por expertos en el área, pero no oficialmente reconocidas y otros cuentan con unos requerimientos de evaluación económica para presentar medicamentos a organismos o entidades de toma de decisiones o para reembolso. En Latinoamérica se cuenta con guías oficiales en Brasil, Colombia, Cuba, México, MERCOSUR (Argentina, Brasil, Paraguay, Uruguay) (ISPOR, 2018). Existe también múltiples publicaciones de esta Sociedad, con lineamientos y conceptos para el desarrollo de evaluaciones económicas y su modelamiento producto de grupos de trabajo (Task Force) e incluso para reporte presentación de informes de estas evaluaciones llamada la CHEERS (Consolidated Health Economic Evaluation Reporting Standards) (Augustovski, García Martí, \& Pichon-Riviere, 2013; Husereau et al., 2013). La Organización Mundial de la Salud cuenta con una guía para realizar evaluaciones de costo-efectividad (TanTorres et al., 2003), e incluso una para estandarización de evaluaciones económicas de programas de inmunización de la OMS (WHO, 2008). 
El grupo de métodos económicos de la colaboración Cochrane señala que la evaluación crítica de los estudios económicos en salud se puede realizar evaluando el riesgo de sesgo y evaluando la calidad metodológica. Para riesgo de sesgo solo consideran las evaluaciones económicas realizadas en conjunto con un estudio de eficacia, enfocando la evaluación de este riesgo en dicho estudio, sin especificar ningún método para las evaluaciones económicas fundamentadas en modelos (Higgins \& Green, 2011).

Para la calidad metodológica de las evaluaciones económicas el grupo Cochrane menciona listas de verificación, como la de chequeo para autores y pares revisores de la British Medical Journal (M. F. Drummond \& Jefferson, 1996), la lista consenso sobre criterios de economía de la salud (Evers, Goossens, de Vet, van Tulder, \& Ament, 2005), las cuales han mostrado evidencia en la confiabilidad entre evaluadores y confiabilidad test-retest, según lo reportado en una revisión sistemática sobre los instrumentos de evaluación de calidad para la realización y presentación de evaluaciones económicas (Walker et al., 2012). Sin embargo, no son suficientes en caso de estudios de modelado, para los que recomiendan (Higgins \& Green, 2011; Shemilt et al., 2013) una lista de la Health Technology Assessment lista de verificación de Philips (Philips et al., 2004) que puede ser complementada con otra que jerarquiza las fuentes usadas para los parámetros en los modelos (Cooper, Coyle, Abrams, Mugford, \& Sutton, 2005).

Posteriormente, en el año 2015 se publicó una lista de chequeo pendiente de validación, llamada "ECOBIAS" que tiene el objetivo de identificar y evaluar sesgos potenciales en evaluaciones económicas (Adarkwah, van Gils, Hiligsmann, \& Evers, 2016). La cual se divide en sesgos generales (perspectiva estrecha, sesgo de patrocinio, etc) y sesgos en el modelo, estos se dividen a su vez en sesgos a nivel de estructura (supuestos estructurales del modelo, elección del tipo de modelo y horizonte temporal, e inclusión de las alternativas posibles), o a nivel de información (como la de entrada en el modelo -eficacia, efectividad, costos-, o la transparencia en el método de incorporación de esta información, como evaluar la incertidumbre), o de consistencia interna del modelo.

Sin embargo, a pesar de contarse con instrumentos para evaluar calidad de evaluaciones económicas, algunos consideran que estos instrumentos presentan debilidades metodológicas (Langer, 2012). Así mismo, se ha considerado que las evaluaciones económicas pueden presentar debilidades metodológicas por falta de unificación de métodos y calidad en las fuentes de información que son usadas para su desarrollo (Pichon-Riviere, Augustovski, Garcia Marti, Sullivan, \& Drummond, 2012), heterogeneidad que dificulta la comparación de resultados (Atehortua, Ceballos, Gaviria, \& Mejia, 2013), o la aplicación de estos estudios para toma de decisiones (Chaikledkaew \& Kittrongsiri, 2014), incluso resaltan falta de difusión de las guias propias o estándar en sus propios contextos para mejorar dichas limitaciones metodológicas (García Fariñas, García Rodríguez, Gálvez González, \& Jimenez López, 2016; Rezapour, Jafari, Mirmasoudi, \& Talebianpour, 2017).

\section{REVISIÓN SISTEMÁTICA DE EVALUACIONES ECONÓMICAS Y SU CALIDAD}

Realizar revisiones sistemáticas de evaluaciones económicas ha sido cuestionado por las limitaciones que se pueden presentar dada la naturaleza de los estudios individuales, con respecto a la fortaleza en la evidencia, por variabilidad en fuentes, uso de recursos, costos, poblaciones, contexto de toma de decisiones, e incluso diferencias dentro de los mismos países (Gomersall et al., 2014, 2015). Lo cual hace improbable poder generar respuestas 
únicas o medidas resumen a las preguntas sobre la relación costo - desenlace, en contraste con las revisiones sistemáticas de ensayos clínicos controlados y aleatorizados donde usualmente se sintetiza la eficacia de intervenciones. Incluso se ha pensado que es poco probable que los meta-análisis en revisiones de evaluaciones de costo efectividad sean significativos (Anderson, 2010).

Algunos consideran que el valor de las revisiones sistemáticas de evaluaciones económicas está dado no por producir un único resultado como en las revisiones de los otros estudios, sino por la información que resumen, como el resultado general de los principales parámetros de las mismas evaluaciones (Drummond MF, 2015), sus rangos, uso de recursos, costos, lo cual puede identificar deficiencias en los estudios o vacíos del conocimiento, que pueden resultar interesantes para investigadores. Así como también esta información puede ayudar a los tomadores de decisiones para comprender la estructura del problema relacionado a la intervención, conocer hallazgos de estudios en diferentes entornos y poblaciones e incluso condiciones en las que la intervención puede ser más eficiente, y que puede facilitar su elección.

En Europa, algunos países cuentan con la directriz de presentar una revisión sistemática de evaluaciones económicas anteriores, cuando se desea realizar una nueva evaluación económica, como en Croacia, Inglaterra, Francia, Polonia, Eslovaquia y España. Incluso la guía de Métodos para evaluaciones económicas en salud de Europa realizada por la "European Network for health technology assessment - EunetHTA", en la cual buscan aumentar la transferibilidad de estos estudios en los países socios, considera útil realizar una revisión sistemática de las evaluaciones anteriores de la tecnología (EunetHTA, 2015). En Latinoamérica en Chile se encuentran desarrollando una guía para transferibilidad de evaluaciones económicas de tecnologías en salud, la cual incluye como primer paso realizar una revisión sistemática de estudios previos para valorar similitudes y ajustes necesarios a realizar (PAHO, 2017; RedETSA, 2017)

Para realizar revisiones sistemáticas de evaluaciones económicas, aún no se encuentra estandarizada una metodología, y se podría considerar lineamientos de diferentes fuentes como: el capítulo 15 del manual Cochrane, en el cual se dan criterios para incorporación de evidencia económica (Higgins \& Green, 2011). Las recomendaciones para revisiones sistemáticas de evaluaciones económicas de la Universidad de York (Akers J et al., 2009), del Instituto Joanna Briggs (Gomersall et al., 2014, 2015), de la revisión sistemática de métodos para revisiones sistemáticas de evaluaciones económicas en salud (Mathes, Walgenbach, Antoine, Pieper, \& Eikermann, 2014), u otras publicaciones que proporcionan información de cómo preparar una revisión sistemática de este tipo de estudios (Thielen et al., 2016; van Mastrigt et al., 2016; Wijnen et al., 2016).

Aunque no se cuente con herramientas estandarizadas para la evaluación de calidad de las evaluaciones económicas al incluirlas en las revisiones sistemáticas e incluso existan detractores para su uso, en lugar de no realizar dicha evaluación, podría considerarse los listados recomendados por el grupo de métodos económicos de Cochrane mencionados en la sección anterior o incluso la nueva herramienta ECOBIAS, para evaluación de riesgo de sesgo, también mencionada anteriormente. El uso del sistema GRADE para calificar la calidad de uso de recursos y de evidencia económica se ha propuesto pero aún no ha presentado suficiente acogida (Brunetti et al., 2013), sin embargo en la última guía de la OMS para toma de decisiones lo consideran dentro de los enfoques para evaluar evidencia económica (Rehfuess et al., 2019). 
Para reporte o evaluación de calidad de revisiones sistemáticas de evaluaciones económicas específicamente, hasta ahora no se cuenta con guías o listas (Mathes et al., 2014). Al no disponerse de una herramienta específica para el reporte y la evaluación metodológica de revisiones sistemáticas de evaluaciones económicas, se podría considerar aplicar tanto PRISMA como AMSTAR, dado que la mayoría de aspectos incluidos en estas herramientas, se basa en los elementos generales que componen una revisión y que son relevantes para todo tipo de revisión sistemática (criterios de elegibilidad, fuentes de información, búsqueda, selección, extracción de datos, resumen de evidencia, síntesis de resultados, etc), aunque aún no existe claridad con el tema de manejo de sesgos de las evaluaciones económicas.

El listado PRISMA ha sido aceptado por ISPOR para guiar la elaboración del informe de las revisiones sistemáticas de evaluaciones económicas, porque muchos de los enunciados aplican directamente, como lo preveían los mismos desarrolladores al considerarlo útil para revisiones sistemáticas de otro tipo de estudios. Sin embargo, sería conveniente que en actualizaciones del listado PRISMA, para uso en revisiones sistemáticas de evaluaciones económicas, se ampliara los ejemplos en algunos enunciados como en la lista de datos o características de los estudios (considerar parámetros de entrada -morbimortalidad, eficacia-efectividad, costos, etc-), en síntesis de datos (tener en cuenta que puede ser en forma narrativa o cualitativa, en gráficas o cuantitativa), y en las medidas resumen (considerar la razón de costo efectividad incremental), lo cual facilitaría el entendimiento por parte de quienes aplican este listado para evaluaciones económicas.

Para la calidad metodológica de las evaluaciones económicas el grupo Cochrane menciona listas de verificación, como la de chequeo para autores y pares revisores de la British Medical Journal (M. F. Drummond \& Jefferson, 1996), la lista consenso sobre criterios de economía de la salud (Evers, Goossens, de Vet, van Tulder, \& Ament, 2005), las cuales han mostrado evidencia en la confiabilidad entre evaluadores y confiabilidad test-retest, según lo reportado en una revisión sistemática sobre los instrumentos de evaluación de calidad para la realización y presentación de evaluaciones económicas (Walker et al., 2012). Sin embargo, no son suficientes en caso de estudios de modelado, para los que recomiendan (Higgins \& Green, 2011; Shemilt et al., 2013) una lista de la Health Technology Assessment lista de verificación de Philips (Philips et al., 2004) que puede ser complementada con otra que jerarquiza las fuentes usadas para los parámetros en los modelos (Cooper, Coyle, Abrams, Mugford, \& Sutton, 2005).

Posteriormente, en el año 2015 se publicó una lista de chequeo pendiente de validación, llamada "ECOBIAS" que tiene el objetivo de identificar y evaluar sesgos potenciales en evaluaciones económicas (Adarkwah, van Gils, Hiligsmann, \& Evers, 2016). La cual se divide en sesgos generales (perspectiva estrecha, sesgo de patrocinio, etc) y sesgos en el modelo, estos se dividen a su vez en sesgos a nivel de estructura (supuestos estructurales del modelo, elección del tipo de modelo y horizonte temporal, e inclusión de las alternativas posibles), o a nivel de información (como la de entrada en el modelo -eficacia, efectividad, costos-, o la transparencia en el método de incorporación de esta información, como evaluar la incertidumbre), o de consistencia interna del modelo.

Sin embargo, a pesar de contarse con instrumentos para evaluar calidad de evaluaciones económicas, algunos consideran que estos instrumentos presentan debilidades metodológicas (Langer, 2012). Así mismo, se ha considerado que las evaluaciones económicas pueden presentar debilidades metodológicas por falta de 
unificación de métodos y calidad en las fuentes de información que son usadas para su desarrollo (Pichon-Riviere, Augustovski, Garcia Marti, Sullivan, \& Drummond, 2012), heterogeneidad que dificulta la comparación de resultados (Atehortua, Ceballos, Gaviria, \& Mejia, 2013), o la aplicación de estos estudios para toma de decisiones (Chaikledkaew \& Kittrongsiri, 2014), incluso resaltan falta de difusión de las guias propias o estándar en sus propios contextos para mejorar dichas limitaciones metodológicas (García Fariñas, García Rodríguez, Gálvez González, \& Jimenez López, 2016; Rezapour, Jafari, Mirmasoudi, \& Talebianpour, 2017).

\section{CONCLUSIONES}

En búsqueda de la mejor evidencia disponible, las revisiones sistemáticas son el tipo de estudios que, con base a su rigurosa metodología, pueden reunir la mejor evidencia, desde la búsqueda de estudios, hasta su integración, síntesis o combinación de hallazgos de los mismos estudios. Sin embargo, no se encuentran exentas de la posibilidad de errores o sesgos, en caso de no seguir estrictamente los procedimientos, aunque también pueden afectar el tipo de estudios que son incluidos.

Existen guías y procedimientos claros, para realizar revisiones sistemáticas de ensayos clínicos controlados y aleatorizados como lo mencionado anteriormente por la Colaboración Cochrane, pero para otro tipo de estudios como en el caso de las evaluaciones económicas, a pesar de las guías existentes para realizar revisiones sistemáticas aún falta estandarización. Adicionalmente por las características de estos estudios con respecto a la variabilidad metodológica y de fuentes de información, resulta más complejo poder sintetizar la información cuando proviene de diferentes contextos.

Las revisiones sistemáticas de evaluaciones económicas deben contemplar lineamientos metodológicos rigurosos siguiendo el modelo de la evolución teórica y metodológica de las revisiones sistemáticas de los ensayos clínicos. Procesos tales como la estrategia de búsqueda de los estudios, el de selección y exclusión, la extracción de información, el análisis y presentación de resultados, asi sea en forma narrativa, podrían ser extrapolados desde los procedimientos de revisiones sistemáticas de los ensayos clínicos. Otros procesos como la evaluación de la calidad metodológica incluyendo el riesgo de sesgo ameritan ser refinados y estandarizados en el caso de las revisiones sistemáticas de evaluaciones económicas. Los listados de reporte de revisiones sistemáticas PRISMA y de metodología AMSTAR, pueden ser considerados al realizar este tipo de revisiones de evaluaciones económicas, porque es posible que ayuden a prevenir debilidades en su desarrollo. Así como también pueden servir para evaluar las revisiones al realizar revisión de revisiones (overview) sistemáticas de evaluaciones económicas.

Las revisiones sistemáticas de evaluaciones económicas, metodológicamente robustas, pueden servir en el complejo proceso de toma de decisiones, al aportar evidencia resumida y confiable, para conocer que estudios económicos se han realizado con la tecnología en salud de interés, que tipo de modelos, fuentes de información epidemiológica y de costos se han utilizado, que supuestos se han empleado, y los resultados que se han encontrado. Información que puede resultar muy útil para tomadores de decisiones a nivel administrativo y de políticas en salud, porque podrían considerar la posibilidad de transferibilidad de estos estudios, realizando los ajustes necesarios y poder evaluar la inclusión de una nueva tecnología o considerar realizar una nueva evaluación económica si esto no es aplicable, como se realiza en algunos países actualmente. 


\section{REFERENCIAS BIBLIOGRÁFICAS}

Adarkwah, C. C., van Gils, P. F., Hiligsmann, M., \& Evers, S. M. (2016). Risk of bias in model-based economic evaluations: the ECOBIAS checklist. Expert Rev Pharmacoecon Outcomes Res, 16(4), 513-523. doi: 10.1586/14737167.2015.1103185

Akers J, Aguiar-Ibáñez R, Baba-Akbari A, Beynon S, Booth A, Burch J, . . Fonseca T. (2009). Systematic Reviews of Economic EvaluationsSystematic Reviews. CRD's guidance for undertaking reviews in health care.: Centre for Reviews and Dissemination, University of York. Retrieved from http://www.york.ac.uk/crd/SysRev/!SSL!/WebHelp/SysRev3.htm.

Anderson, R. (2010). Systematic reviews of economic evaluations: utility or futility? Health Econ, 19(3), 350-364. doi: 10.1002/hec.1486

Atehortua, S., Ceballos, M., Gaviria, C. F., \& Mejia, A. (2013). [Quality assessment of economic evaluations in health care in Colombia: a systematic review]. Biomedica, 33(4), 615-630.

Atkins, D., Best, D., Briss, P. A., Eccles, M., Falck-Ytter, Y., Flottorp, S., .. . Zaza, S. (2004). Grading quality of evidence and strength of recommendations. BMJ, 328(7454), 1490. doi:10.1136/ bmj.328.7454.1490

Augustovski, F., García Martí, S., \& Pichon-Riviere, A. (2013). Estándares Consolidados de Reporte de Evaluaciones Económicas Sanitarias: Versión en Español de la Lista de Comprobación CHEERS. Value in Health Regional Issues, 2(3), 338-341. doi: http://dx.doi.org/10.1016/j. vhri.2013.10.004

Borgerson, K. (2009). Valuing evidence: bias and the evidence hierarchy of evidence-based medicine. Perspect Biol Med, 52(2), 218-233. doi: 10.1353/pbm.0.0086

Briggs A, C. K., Sculper M,. (2007). Decision modelling for health economic evaluation. Oxford UK: Oxford University Press.

Brouwer, W. B., Culyer, A. J., van Exel, N. J., \& Rutten, F. F. (2008). Welfarism vs. extra-welfarism. J Health Econ, 27(2), 325-338. doi: 10.1016/j.jhealeco.2007.07.003

Brunetti, M., Shemilt, I., Pregno, S., Vale, L., Oxman, A. D., Lord, J., .. Schunemann, H. J. (2013). GRADE guidelines: 10 . Considering resource use and rating the quality of economic evidence. J Clin Epidemiol, 66(2), 140-150. doi: 10.1016/j.jclinepi.2012.04.012

Cochrane Website. Cochrane Collaboration. Retrieved Marzo 7, 2019, from https://www.cochrane. org/about-us

Cooper, N., Coyle, D., Abrams, K., Mugford, M., \& Sutton, A. (2005). Use of evidence in decision models: an appraisal of health technology assessments in the UK since 1997. J Health Serv Res Policy, 10(4), 245-250. doi: 10.1258/135581905774414187

Chaikledkaew, U., \& Kittrongsiri, K. (2014). Quality assessment of health economic evaluation. J Med Assoc Thai, 97 Suppl 5, S113-118.

Drummond, M., Barbieri, M., Cook, J., Glick, H. A., Lis, J., Malik, F., ... Severens, J. (2009). Transferability of economic evaluations across jurisdictions: ISPOR Good Research Practices Task Force report. Value Health, 12(4), 409-418. doi: 10.1111/j.1524-4733.2008.00489.x

Drummond, M. F., \& Jefferson, T. O. (1996). Guidelines for authors and peer reviewers of economic submissions to the BMJ. The BMJ Economic Evaluation Working Party. BMJ, 313(7052), 275-283. 
Drummond MF, S. M., Claxton K, Stoddart GL, Torrance GW (2015). Methods for the Economic Evaluation of Health Care Programmes. Oxford - United Kingdom.

EunetHTA. (2015). Methods for health economic evaluations - A guideline based on current practices in Europe. Retrieved Marzo 23, 2019, from https://www.eunethta.eu/wpcontent/uploads/2018/03/Methods_for_health_economic_evaluations.pdf

Evers, S., Goossens, M., de Vet, H., van Tulder, M., \& Ament, A. (2005). Criteria list for assessment of methodological quality of economic evaluations: Consensus on Health Economic Criteria. Int J Technol Assess Health Care, 21(2), 240-245.

García Fariñas, A., García Rodríguez, J. F., Gálvez González, A. M., \& Jimenez López, G. (2016). Calidad metodológica de las evaluaciones económicas completas, publicadas en revistas médicas cubanas (1999-2014). Revista Cubana de Salud Pública, 42, 183-192.

Gomersall, J. S., Jadotte, Y. T., Xue, Y., Lockwood, S., Riddle, D., \& Preda, A. (2014). The Systematic Review of Economic Evaluation Evidence. : The Joanna Briggs Institute Reviewers' Manual 2014.

Gomersall, J. S., Jadotte, Y. T., Xue, Y., Lockwood, S., Riddle, D., \& Preda, A. (2015). Conducting systematic reviews of economic evaluations. Int J Evid Based Healthc, 13(3), 170-178. doi: $10.1097 /$ xeb.0000000000000063

Gray, A. M. C., P.M.; Wolstenholme, J.L.; Wordsworth S. (2011). Applied Methods of Cost-effectiveness Analysis in Health Care. Oxford United Kingdom: Oxford University Press.

Herner, M. (2019). Perfect Top of the Evidence Hierarchy Pyramid, Maybe Not So Perfect: lessons learned by a novice researcher engaging in a meta-analysis project. BMJ Evid Based Med. doi: 10.1136/bmjebm-2018-111141

Higgins, J., \& Green, S. (Producer). (2011). Cochrane Handbook for Systematic Reviews of Interventions Version 5.1.0. Retrieved from www.cochrane-handbook.org.

Howick, J., Chalmers, L., Glasziou, P., Greenhalgh, T., Heneghan, C., Liberati, A., . . Hodgkinson, M. (2011). The Oxford Levels of Evidence 2. Retrieved Marzo 12, 2019, from https://www. cebm.net/index.aspx?o=5653

Husereau, D., Drummond, M., Petrou, S., Carswell, C., Moher, D., Greenberg, D., . . Loder, E. (2013). Consolidated Health Economic Evaluation Reporting Standards (CHEERS)-explanation and elaboration: a report of the ISPOR Health Economic Evaluation Publication Guidelines Good Reporting Practices Task Force. Value Health, 16(2), 231-250. doi: 10.1016/j.jval.2013.02.002

INHATA. The International Network of Agencies for Health Technology Assessment. Retrieved Marzo 5, 2019, from http://www.inahta.org/

ISPOR. (2018). Pharmacoeconomic Guidelines Around the World. Retrieved Marzo 18, 2019 from https://tools.ispor.org/peguidelines/

Langer, A. (2012). A framework for assessing Health Economic Evaluation (HEE) quality appraisal instruments. BMC Health Serv Res, 12, 253. doi: 10.1186/1472-6963-12-253

Liberati, A., Altman, D. G., Tetzlaff, J., Mulrow, C., Gotzsche, P. C., Ioannidis, J. P., . . Moher, D. (2009). The PRISMA statement for reporting systematic reviews and meta-analyses of studies that evaluate health care interventions: explanation and elaboration. Plos Med, 6(7), e1000100. doi: 10.1371/journal.pmed.1000100 
Luhnen, M., Prediger, B., Neugebauer, E. A. M., \& Mathes, T. (2019). Systematic reviews of health economic evaluations: A structured analysis of characteristics and methods applied. doi: 10.1002/jrsm.1342

Manterola, C., Asenjo-Lobos, C., \& Otzen, T. (2014). [Hierarchy of evidence: levels of evidence and grades of recommendation from current use]. Rev Chilena Infectol, 31(6), 705-718. doi: $10.4067 / \mathrm{s} 0716-10182014000600011$

Mathes, T., Walgenbach, M., Antoine, S. L., Pieper, D., \& Eikermann, M. (2014). Methods for systematic reviews of health economic evaluations: a systematic review, comparison, and synthesis of method literature. Med Decis Making, 34(7), 826-840. doi: 10.1177/0272989x14526470

Murad, M. H., Asi, N., Alsawas, M., \& Alahdab, F. (2016). New evidence pyramid. 21(4), 125-127. doi: 10.1136/ebmed-2016-110401

Ng, Y. K. (2004). Welfare Economics: Towards a More complete Analysis. New York - USA: Palgrave Macmillan.

PAHO, T. V. (Producer). (2017, Marzo 20 de 2019). Webinar Transferibilidad de evaluaciones económicas. Retrieved from https://www.youtube.com/watch?v=oE1N35d5mEw

Philips, Z., Ginnelly, L., Sculpher, M., Claxton, K., Golder, S., Riemsma, R., . . Glanville, J. (2004). Review of guidelines for good practice in decision-analytic modelling in health technology assessment. Health Technol Assess, 8(36), iii-iv, ix-xi, 1-158.

Pichon-Riviere, A., Augustovski, F., Garcia Marti, S., Sullivan, S. D., \& Drummond, M. (2012). Transferability of health technology assessment reports in Latin America: an exploratory survey of researchers and decision makers. Int J Technol Assess Health Care, 28(2), 180186. doi: $10.1017 / \mathrm{s} 0266462312000074$

Pieper, D., Koensgen, N., Breuing, J., Ge, L., \& Wegewitz, U. (2018). How is AMSTAR applied by authors - a call for better reporting. BMC Med Res Methodol, 18(1), 56. doi: 10.1186/s12874018-0520-z

Pollock, M., Fernandes, R. M., Becker, L. A., Featherstone, R., \& Hartling, L. (2016). What guidance is available for researchers conducting overviews of reviews of healthcare interventions? A scoping review and qualitative metasummary. Syst Rev, 5(1), 190. doi: 10.1186/s13643016-0367-5

Pollock, M., Fernandes, R. M., \& Hartling, L. (2017). Evaluation of AMSTAR to assess the methodological quality of systematic reviews in overviews of reviews of healthcare interventions. BMC Med Res Methodol, 17(1), 48. doi: 10.1186/s12874-017-0325-5

RedETSA (Producer). (2017, Marzo 18 2019). Transferibilidad de evaluaciones económicas. Retrieved from http://redetsa.org/wp/?p=3994

Rehfuess, E. A., Stratil, J. M., Scheel, I. B., Portela, A., Norris, S. L., \& Baltussen, R. (2019). The WHOINTEGRATE evidence to decision framework version 1.0: integrating WHO norms and values and a complexity perspective. BMJ Glob Health, 4(Suppl 1), e000844. doi: 10.1136/bmjgh-2018-000844

Rezapour, A., Jafari, A., Mirmasoudi, K., \& Talebianpour, H. (2017). Quality Assessment of Published Articles in Iranian Journals Related to Economic Evaluation in Health Care Programs Based on Drummond's Checklist: A Narrative Review. Iran J Med Sci, 42(5), 427-436.

Sackett, D. L., Rosenberg, W. M., Gray, J. A., Haynes, R. B., \& Richardson, W. S. (1996). Evidence based medicine: what it is and what it isn't. BMJ, 312, 71-72. 
Shea, B. J., Grimshaw, J. M., Wells, G. A., Boers, M., Andersson, N., Hamel, C., . . Bouter, L. M. (2007). Development of AMSTAR: a measurement tool to assess the methodological quality of systematic reviews. BMC Med Res Methodol, 7, 10. doi: 10.1186/1471-2288-7-10

Shea, B. J., Hamel, C., Wells, G. A., Bouter, L. M., Kristjansson, E., Grimshaw, J., . . Boers, M. (2009). AMSTAR is a reliable and valid measurement tool to assess the methodological quality of systematic reviews. J Clin Epidemiol, 62(10), 1013-1020. doi: 10.1016/j.jclinepi.2008.10.009

Shea, B. J., Reeves, B. C., Wells, G., Thuku, M., Hamel, C., Moran, J., ... Henry, D. A. (2017). AMSTAR 2: a critical appraisal tool for systematic reviews that include randomised or nonrandomised studies of healthcare interventions, or both. BMJ, 358, j4008. doi: 10.1136/ bmj.j4008

Shemilt, I., McDaid, D., Marsh, K., Henderson, C., Bertranou, E., Mallander, J., . . Vale, L. (2013). Issues in the incorporation of economic perspectives and evidence into Cochrane reviews. Syst Rev, 2, 83. doi: 10.1186/2046-4053-2-83

SIGN, G. (2011). SIGN 50 A guideline developer's handbook. Scottish Intercollegiate Guidelines Network SIGN Retrieved from https://www.sign.ac.uk/assets/sign50_2011.pdf.

Smith, V., Devane, D., Begley, C. M., \& Clarke, M. (2011). Methodology in conducting a systematic review of systematic reviews of healthcare interventions. BMC Med Res Methodol, 11(1), 15. doi: 10.1186/1471-2288-11-15

Soto-Alvarez, J. (2012). Evaluación económica de medicamentos y tecnologías sanitarias: Principios, métodos y aplicaciones en política sanitaria. (S. A. U. Springer SBM Spain Ed.).

Madrid, España.

Tan-Torres, E., Baltusen, R., Adam, T., Hutubessy, R., Acharya, A., Evans, D., \& Murray, C. J. L. (2003). Making choices in health: WHO Guide to Cost-effectiveness Analysis. from https://www. who.int/choice/publications/p_2003_generalised_cea.pdf

Thielen, F. W., Van Mastrigt, G., Burgers, L. T., Bramer, W. M., Majoie, H., Evers, S., \& Kleijnen, J. (2016). How to prepare a systematic review of economic evaluations for clinical practice guidelines: database selection and search strategy development (part 2/3). Expert Rev Pharmacoecon Outcomes Res, 16(6), 705-721. doi: 10.1080/14737167.2016.1246962

Thulliez, M., Angoulvant, D., Pisella, P. J., \& Bejan-Angoulvant, T. (2018). Overview of Systematic Reviews and Meta-analyses on Systemic Adverse Events Associated With Intravitreal Anti-Vascular Endothelial Growth Factor Medication Use. JAMA Ophthalmol, 136(5), 557-566. doi: 10.1001/jamaophthalmol.2018.0002

Urrutia, G., \& Bonfill, X. (2010). [PRISMA declaration: a proposal to improve the publication of systematic reviews and meta-analyses]. Med Clin (Barc), 135(11), 507-511. doi: 10.1016/j. medcli.2010.01.015

van Mastrigt, G. A., Hiligsmann, M., Arts, J. J., Broos, P. H., Kleijnen, J., Evers, S. M., \& Majoie, M. H. (2016). How to prepare a systematic review of economic evaluations for informing evidence-based healthcare decisions: a five-step approach (part 1/3). Expert Rev Pharmacoecon Outcomes Res, 16(6), 689-704. doi: 10.1080/14737167.2016.1246960

Walker, D. G., Wilson, R. F., Sharma, R., Bridges, J., Niessen, L., Bass, E. B., \& Frick, K. (2012). Best Practices for Conducting Economic Evaluations in Health Care: A Systematic Review of Quality Assessment Tools: The Johns Hopkins University Evidence-based Practice Center. 
WHO, W. H. O. (2008). WHO guide for standardization of economic evaluations of immunization programmes (V. a. B. Department of Immunization, Trans.). Geneva, Switzerland: World Health Organization.

Wijnen, B., Van Mastrigt, G., Redekop, W. K., Majoie, H., De Kinderen, R., \& Evers, S. (2016). How to prepare a systematic review of economic evaluations for informing evidence-based healthcare decisions: data extraction, risk of bias, and transferability (part 3/3). Expert Rev Pharmacoecon Outcomes Res, 16(6), 723-732. doi: 10.1080/14737167.2016.1246961 